\title{
Introduction to the HICSS-55 Minitrack on Geospatial Big Data Analytics
}

\author{
Joseph Aversa \\ Ted Rogers School \\ of Management, \\ Ryerson University \\ javersa@ryerson.ca
}

\section{Introduction}

Geographic perspectives have historically contributed unique solutions to diverse problems, centred on the complex relationships between humans and their environments. With increases in sensors, social media, human mobility data, spatial imagery and remote sensing, geospatial big data presents an emerging frontier of research and innovation in the system sciences and across various sectors and industries. Advancements in geospatial big data have provided businesses and organizations with an ability to obtain more granular level information on human behaviour.

With the increased automation of data collection and analysis, along with the development of algorithms that can extract and illustrate patterns in human behaviours, geospatial big data has raised key questions about research processes and the ways in which academics and industry practitioners should engage with these data sources. There is an ever growing need to address questions on how to make sense of these vast amounts of raw information and how to evaluate the role of traditional forms of scientific theories and models in assessing these types of data. With geospatial big data providing immense opportunity for expanding our understanding of human behaviours, there has been increased reliance on new data sources for understanding human interaction across many disciplines.

This minitrack includes papers that analyze and mine geospatial big data using cutting-edge scientific approaches to provide insights to complex problems and systems in business, government, and society. This minitrack includes both theoretical and empirical papers in a variety of analytics contexts including mobile analytics, consumer decision-making and business decision support. The following provides a brief overview of the three papers included in this minitrack.

\section{Geo-Located transaction data}

The paper on "Tracking the Impact of the Covid-19 Pandemic with the Use of High-Frequency Geo-Located Bank Transaction Data" by N. Neuteboom, G.
Kapetanios, A. Ventouri, and F. Ritsema, analyses the economic effects of consumer responses to Covid-19 during different waves of the pandemic. Using geolocated transaction data, from 2 million customers of ABN AMRO bank in the Netherlands, the paper compares municipalities that experienced large Covid-19 outbreaks with municipalities that had few or no cases. The study finds that during the first wave of the pandemic, the scale of the outbreak in a municipality had a strong negative effect on physical transactions by consumers. This behavioral response function of consumers towards the virus was not constant over time as the behavioural effect of consumers towards the virus during the second wave of infection had no real impact on consumption patterns. By geo-locating transactions by municipality, this study was able to identify the consumer response to Covid-19.

\section{Mobile location data}

The paper on "The effect of COVID-19 on customer traffic: A case study of Food and Beverage stores in Erie County, New York" by J.Aversa, X, Quach, T. Hernadez, $R$. Vatrapu investigates the pressures placed on retailers as a result of the restrictive government policies that were put into place to curb the spread of the COVID-19 virus. Using a case study of Erie County in the State of New York (NY), the paper investigates the changes in visitation patterns for a commercial service sector that was deemed 'essential' - food and beverage. Using mobile data for 602 food and beverage store locations, this research identifies variations in shopping patterns for independent and chain stores. Furthermore, the study finds that by comparing data pre-pandemic and during the pandemic, there were notable changes to visitation patterns over time and between retail types.

\section{GIS Implementation - business Decision- Support}

The paper on "Building GIS Platforms for Spatial Business: A Focus on the Science of Maximizing 
Location Intelligence Benefits Through Risk/Cost Management" by C. Franklin, J. Sreedharan presents an innovative approach to the pursuit of a robust GIS implementation, to contribute to a stronger foundation for optimizing long-term location intelligence benefits for businesses. The paper argues that management practitioners do not estimate risk with the required quantitative rigor during the planning phases, leading to flawed decision models. For organizations competing in a global economy, strong decision models lead to management decision-making options in the creation of value from location awareness and proximity intelligence capabilities. This paper highlights the need for rigorous risk analysis during the planning, and operational phases of GIS implementation to optimize location awareness.

\section{Conclusion}

Geospatial big data promises to offer comprehensive, interconnected, spatiotemporal data that can provide an increased level of sophistication and a more detailed understanding of human behavior. Furthermore, it presents the possibility of a new era of decision making centered on data-driven decisions that will challenge the boundaries and nature of location intelligence. The contributors to this inaugural research minitrack have showcased a number of research strands in which novel data sources and methodologies can play a key role for industry practitioners and academics. 